\title{
DINF: Uma Plataforma Online para Termos da Área de Informática em Libras
}

\author{
Geycy D. O. Lima1, Rafael D. Araújoº \\ ${ }^{1}$ Instituto Federal de Educação, Ciência e Tecnologia de Mato Grosso \\ Campus Pontes e Lacerda \\ ${ }^{2}$ Faculdade de Computação - Universidade Federal de Uberlândia \\ Campus Monte Carmelo \\ geycy.lima@plc.ifmt.edu.br, rafael.araujo@ufu.br
}

\begin{abstract}
Inclusive education has often been discussed in order to create support tools through technological and educational innovation. Especially in fields with many foreign language terms, literal translation of words can lead to misinterpretations. Thus, this work aims at developing a collaborative Webbased platform for presenting a contextualized dictionary of informatics terms in the Brazilian Language of Signals. Several words were mapped out and edited videos with their translation were made available in an institutional domain linked to a federal teaching institution, where there are many deaf students.
\end{abstract}

Resumo. O ensino inclusivo tem sido discutido com frequência para se criar ferramentas de apoio por meio da inovação tecnológica e educacional. Especialmente em áreas com muitos termos em língua estrangeira, a tradução literal das palavras pode levar a interpretações errôneas. Assim, esse trabalho objetiva desenvolver uma plataforma Web colaborativa para a apresentação de um dicionário contextualizado para o ensino de termos relacionados com a área de informática na Língua Brasileira de Sinais. Diversas palavras já foram mapeadas e vídeos editados com sua tradução foram disponibilizados em um domínio institucional vinculado a uma instituição federal de ensino, que possui uma grande quantidade de estudantes surdos.

\section{Introdução}

Existem diversos aplicativos de tradução para a Língua Brasileira de Sinais (Libras), em sua maioria com apenas uma tradução simples e literal, sem explicação de cada conceito. Para quem não sabe Libras, uma simples tradução de um termo qualquer sem entender seu contexto pode gerar confusão e até mesmo um equívoco com relação ao conceito envolvido. Além de já existirem problemas com palavras ambíguas na Língua Portuguesa, a característica contextual de algumas palavras se torna um fator agravante.

Sabe-se que na área da informática, a maioria de seus termos tem origem da Língua Inglesa e que seus conceitos não condizem com a tradução literal da palavra para a Língua Portuguesa e/ou siglas relacionadas. Com isso, tradutores de libras para surdos podem enfrentar problemas em sua interpretação. Santos, Coutinho, \& Brito (2016) listaram 28 ferramentas disponíveis para o ensino de Libras, porém o estudo não relata nenhuma ferramenta específica para o ensino de informática. 
VII Congresso Brasileiro de Informática na Educação (CBIE 2018)

Anais do XXIV Workshop de Informática na Escola (WIE 2018)

A Lei 10.436 de 24 de abril de 2002, diz em seu artigo $2^{\circ}$, que deve ser garantido, por parte do poder público em geral e empresas concessionárias de serviços públicos, formas institucionalizadas de apoiar o uso e a difusão de Libras como meio de comunicação objetiva e de utilização corrente das comunidades surdas do Brasil [Brasil 2002].

De acordo com i.Social [i.Social 2017], estimativas da Organização das Nações Unidas (ONU) indicam que existem cerca de 610 milhões de indivíduos com alguma limitação em todo o mundo. Só no Brasil, país considerado um dos campeões em população com deficiência, $23,9 \%$ da sua população apresenta algum tipo de deficiência, seja visual, auditiva, motora e mental ou intelectual [Oliveira 2012]. Segundo dados do Censo 2010 do IBGE, no Brasil, 18,6\% do total de pessoas com deficiência possuem deficiência visual, 7\% motora, 1,4\% mental ou intelectual e 5,1\% deficiência auditiva, conforme ilustra o Gráfico 1 .

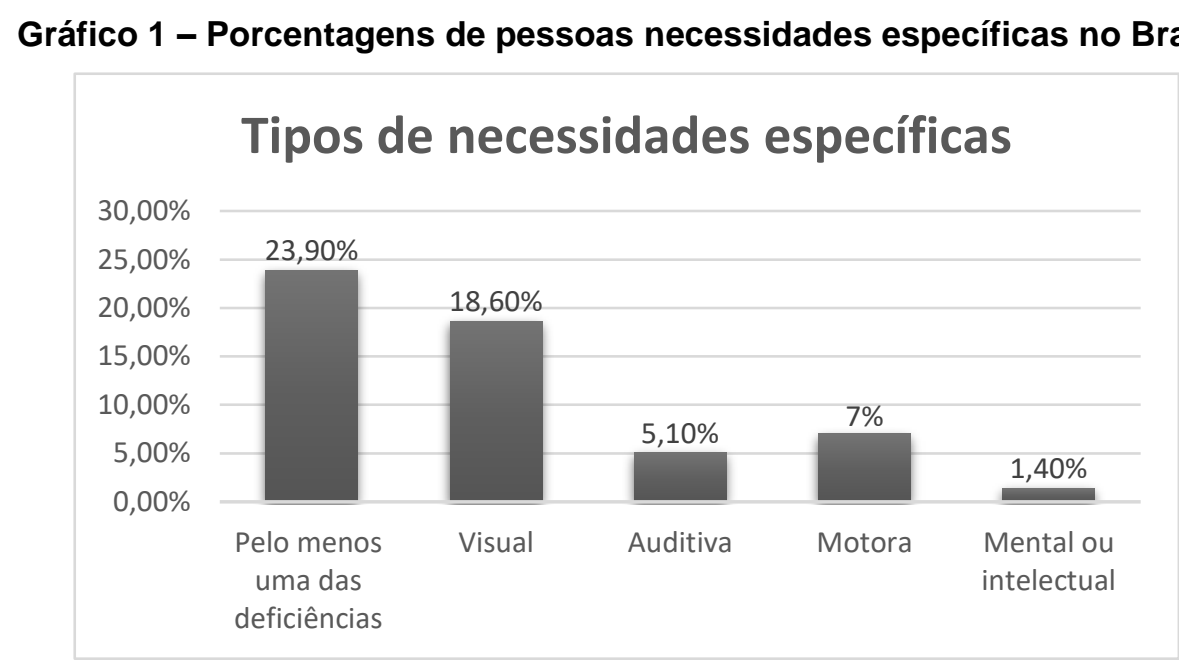

Fonte: Adaptado de [Oliveira, 2012].

A escola tem um papel social de incluir os estudantes com qualquer tipo de necessidade específica. Para tanto, é necessário que os professores, técnicos e intérpretes trabalhem de forma homogênea para que possa ser realizado um bom trabalho e, assim, ofertar melhores experiências para os estudantes.

Pensando em uma maneira de facilitar a compreensão dos termos e conceitos relacionados com a área de informática na Língua Brasileira de Sinais (Libras), propõese nesse trabalho o desenvolvimento de uma plataforma Web para apresentação de um dicionário de termos de informática em Libras para estudantes, professores e profissionais da área.

Com isso, espera-se que a plataforma auxilie a aprendizagem de estudantes da área de informática que possuem necessidades específicas de audição. Disponibilizada de forma gratuita na Internet, pretende-se expandir o trabalho para contemplar termos de diferentes disciplinas da mesma área e avaliar a utilização e a usabilidade da plataforma com seu público-alvo. 
VII Congresso Brasileiro de Informática na Educação (CBIE 2018)

Anais do XXIV Workshop de Informática na Escola (WIE 2018)

O restante do artigo está estruturado como segue: a Seção 2 apresenta uma discussão dos trabalhos relacionados; a Seção 3 expõe a metodologia de pesquisa utilizada nesse projeto; a Seção 4 apresenta as discussões preliminares relacionadas com o desenvolvimento e utilização da plataforma; e, por fim, as considerações finais são apresentadas na Seção 5.

\section{Trabalhos Relacionados}

As tecnologias assistivas são mediadoras na construção do conhecimento, da subjetivação e da autonomia. Diversas ferramentas surgiram para auxiliar a aprendizagem de pessoas com alguma necessidade específica. Para os surdos despontaram o VLibras, ProDeaf e HandTalk. Todas essas aplicações são utilizadas para a tradução automática da Língua Portuguesa para Libras.

O VLibras, por exemplo, é uma suíte de ferramentas muito utilizada para a tradução de diversos termos da Língua Portuguesa para Língua Brasileira de Sinais (Libras). Tais ferramentas são de código aberto e foram desenvolvidas por meio de uma parceria entre o Ministério do Planejamento, Desenvolvimento e Gestão, por meio da Secretaria de Tecnologia da Informação e a Universidade Federal da Paraíba [VLibras 2018]. O VLibras é uma ferramenta que realiza a tradução dos termos de forma automática, por meio de agentes animados $3 \mathrm{D}$, sob a condição de intérpretes virtuais. Entretanto, a aplicação possui um dicionário de sinais da língua portuguesa. Alguns termos de informática da língua inglesa, por exemplo, são apenas soletrados.

O ProDeaf é uma ferramenta móvel para tradução de texto e voz na língua portuguesa para a Língua Brasileira de Sinais, com o objetivo de realizar a comunicação entre surdos e ouvintes [ProDeaf 2018]. O software foi desenvolvido na Universidade Federal de Pernambuco e está disponível para os sistemas iOS, Android e Windows Phone. O aplicativo funciona pela inclusão do texto em português ou através da ferramenta de reconhecimento de voz. O resultado da tradução é uma animação em 3D com os sinais.

Outra aplicação utilizada para a tradução automática é o Hand Talk. A plataforma foi desenvolvida por uma startup alagoana, com intuito de tornar a comunicação mais acessível [Hand Talk 2018]. A ferramenta é baseada em um personagem virtual chamado Hugo (uma animação em 3D), que realiza a tradução das palavras em português para Libras.

Os autores Corrêa et. al (2014), apresentaram em um estudo, as principais fragilidades encontradas no ProDeaf e no Hand Talk. Dentre as fragilidades citadas, destacam-se: dicionário de sinais restrito, dificuldades técnicas quanto ao uso das ferramentas, ausência de expressão facial/corporal e traduções de alguns termos incorretas.

Basicamente, a grande diferença entre os trabalhos citados e a proposta aqui apresentada está na forma com que a tradução para Libras é feita. Enquanto uma tradução automática entre idiomas não considera o contexto de utilização da palavra, este trabalho está focado no contexto de informática. Além disso, considerando aspectos tecnológicos, este trabalho se encontra disponível apenas para Web enquanto os outros trabalhos relacionados apresentam versões móveis de seus aplicativos. 
VII Congresso Brasileiro de Informática na Educação (CBIE 2018)

Anais do XXIV Workshop de Informática na Escola (WIE 2018)

\section{Metodologia}

Esse projeto foi idealizado e tem sido desenvolvido no âmbito dos laboratórios de uma instituição federal de ensino básico e tecnológico, com auxílio de estudantes, professores de informática e especialistas tradutores de Libras. A plataforma criada foi hospedada em um domínio institucional ${ }^{1}$ para melhor difusão da ferramenta entre os estudantes da instituição em questão, uma vez que a mesma possui um histórico de estudantes com necessidades específicas matriculadas em seus vários níveis escolares que contemplam disciplinas da área de informática.

A plataforma foi nomeada de DINF como acrônimo para "Dicionário de Informática em Libras". Sua construção foi apoiada pelo sistema de gerenciamento de conteúdo WordPress. O WordPress é um software gratuito e disponível para qualquer usuário para a criação de sites, escrito em PHP e com banco de dados MySQL [WordPress 2017]. A plataforma criada possui uma interface de uso simples que contempla apenas um campo para a busca da palavra desejada e alguns botões de compartilhamento para incentivar a divulgação do DINF no círculo social do estudante, disponível para diversas redes sociais, como Facebook, Twitter, WhatsApp, dentre outras.

A Figura 1 mostra a metodologia utilizada no desenvolvimento do projeto, que foi dividida em duas fases. É importante salientar que um levantamento bibliográfico sobre o ensino de Libras, aplicações existentes do mercado e os termos de informática foi contemplado em todas as etapas previstas da primeira fase.

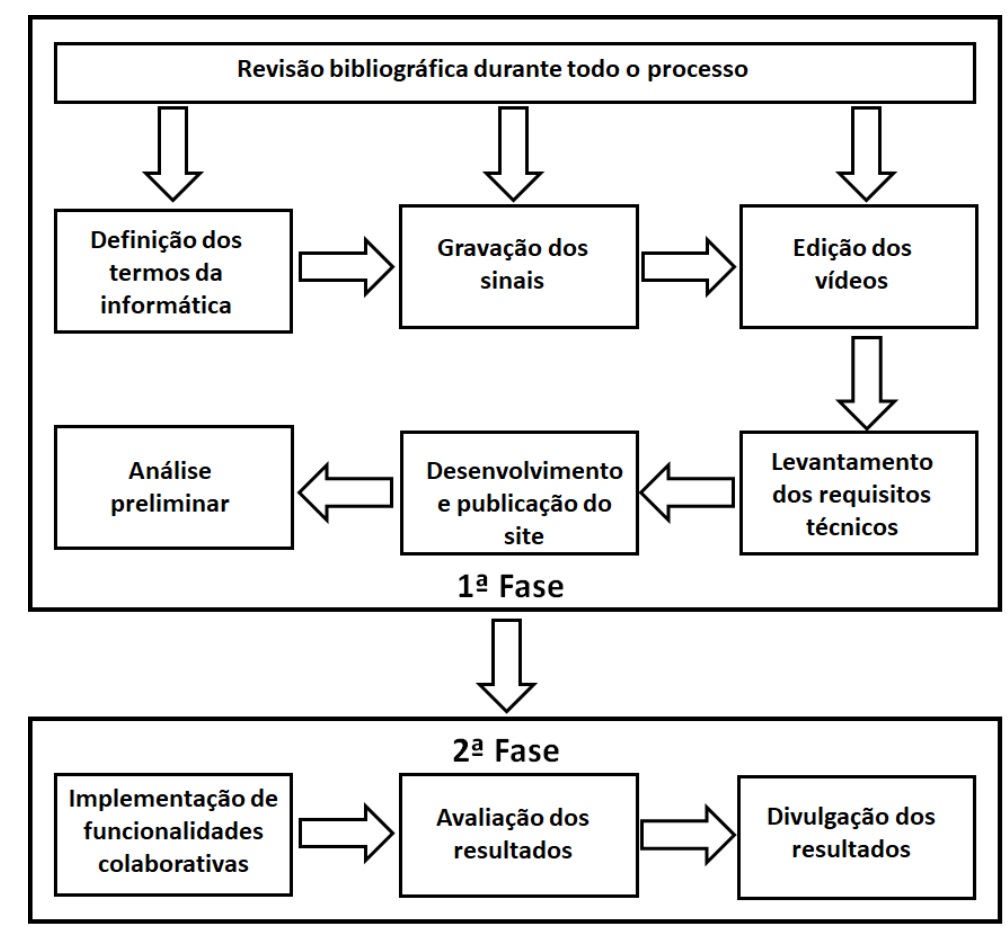

Figura 1 - Metodologia aplicada.

\footnotetext{
${ }^{1}$ http://dinf.plc.ifmt.edu.br
} 
VII Congresso Brasileiro de Informática na Educação (CBIE 2018)

Anais do XXIV Workshop de Informática na Escola (WIE 2018)

\subsection{Primeira fase}

Em um primeiro momento, 287 palavras foram levantadas por estudantes, professores de cursos da área de informática e professores/intérpretes de Libras no projeto de pesquisa intitulado de "Construção de um dicionário dos termos de informática em Libras". Uma vez definidas as palavras, os sinais correspondentes foram gravados por um colaborador surdo em um estúdio de gravação profissional - Centro de Capacitação de Profissionais da Educação e Atendimento às Pessoas com Surdez (CAS) da cidade de Montes Claros/MG - acompanhado por um professor de Libras.

Em seguida, os vídeos foram editados para garantir qualidade e legibilidade antes de serem disponibilizados. Além disso, foi realizada uma etapa de levantamento de requisitos técnicos para identificar as tecnologias necessárias para suportar a disponibilização do dicionário proposto. Quando implementada e com os vídeos disponibilizados, foi realizada uma avaliação preliminar com especialistas da área de tradução em Libras e professores de informática para verificar os elementos qualitativos relacionados com a facilidade de uso e a percepção de utilidade da plataforma bem como o nível de conformidade das palavras traduzidas. Essa etapa serviu para identificar pontos de melhorias na proposta.

\subsection{Segunda fase}

Após uma análise preliminar sobre a utilização da plataforma, iniciou-se a segunda fase da metodologia, conforme Figura 1. Nesse momento, iniciou-se a implementação de funcionalidades colaborativas para a plataforma que visa torna-la autossustentável no futuro, ou seja, sem haver a necessidade da existência de mediadores para manutenção do dicionário.

A primeira funcionalidade é a criação de um campo para envio de sugestões de inclusão de novos termos pelos usuários (estudantes e professores). Tais sugestões serão gravadas no banco de dados da plataforma para que possam ser traduzidas em um segundo momento e, então, disponibilizadas no formato de vídeo.

A segunda funcionalidade colaborativa está relacionada com a gravação dos sinais correspondentes aos termos do dicionário pelos próprios usuários que poderão enviá-los para uma área temporária antes de serem incluídos oficialmente no dicionário. Os vídeos devem ser gravados seguindo algumas regras como, por exemplo, duração, formato e qualidade de imagem. Estudantes e professores podem acessar esses vídeos não-oficiais e indicar a qualidade da tradução dos mesmos (utilizando o mecanismo de estrelas) para que possa ser criado um ranking de vídeos para os sinais gravados. Aqueles que possuírem mais de cinco avaliações com média superior à 4 pontos serão incluídos no dicionário.

Uma vez publicada na Internet, torna-se necessário uma etapa de divulgação da plataforma para atrair estudantes tanto de ensino médio (16 a 18 anos) quanto estudantes do ensino superior (19 a 50 anos), bem como professores e pessoas em geral que tenham interesse em aprender os conceitos relacionados com a área de informática em Libras. Pretende-se coletar dados de utilização da plataforma para avaliar os resultados com relação à comportamento de uso e também com questionários relacionados à experiência do usuário a cada seis meses de sua publicação. 
VII Congresso Brasileiro de Informática na Educação (CBIE 2018)

Anais do XXIV Workshop de Informática na Escola (WIE 2018)

\section{Discussão}

Até o momento, o dicionário contempla um total de 287 termos traduzidos para Libras com vídeos dos respectivos sinais já editados profissionalmente e disponibilizados de forma online para toda a comunidade brasileira. A Figura 2 (a) mostra a página inicial da plataforma DINF e a Figura 2 (b) mostra a exibição de um vídeo com a tradução do termo "computador".

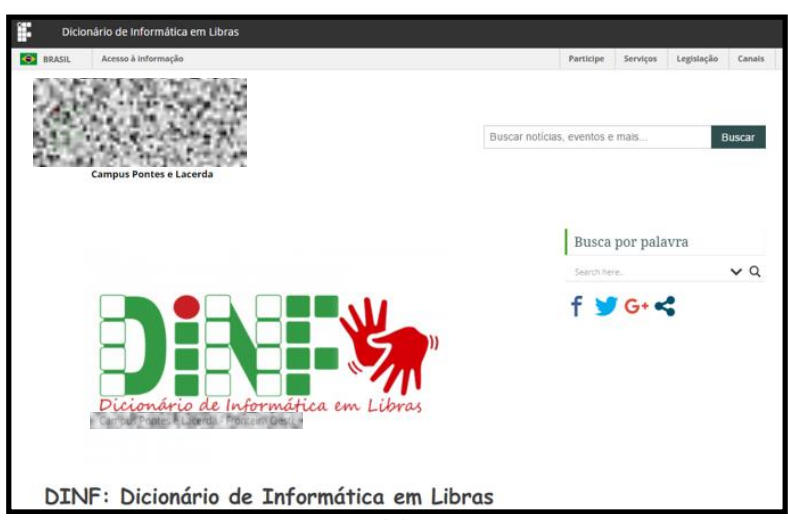

(a)

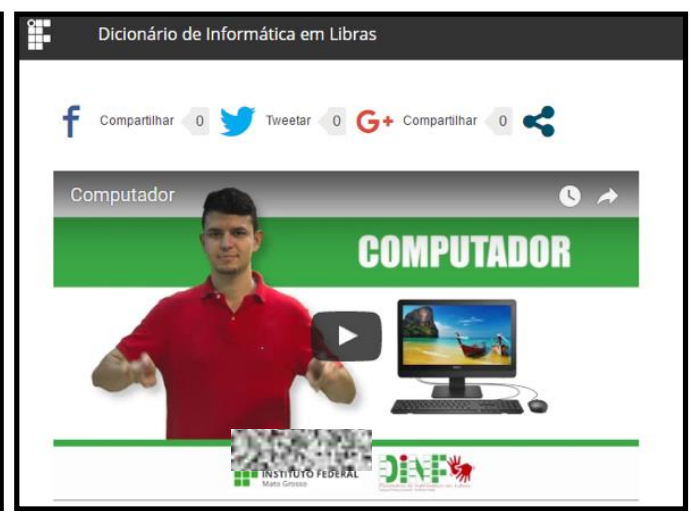

(b)

Figura 2 - Plataforma do Dicionário de Informática em Libras.

Várias pesquisas encontradas na literatura já evidenciaram fragilidades nas traduções automatizadas realizadas por aplicativos [Corrêa, Gomes \& Ribeiro 2017]. Não só traduções de palavras ambíguas como significado de termos fora de um contexto leva a um entendimento incorreto por parte dos usuários das ferramentas de tradução.

Exemplificando, na área de informática, muitas palavras apresentam traduções ambíguas. A palavra "bug", por exemplo, significa um problema ou erro no software e a sua tradução literal do Inglês é inseto/besouro. Outro exemplo, o termo "mouse" contextualizado na área significa um "periférico/dispositivo de entrada do computador" e sua tradução literal seria rato (animal). Da mesma forma, existem vários outros termos que apresentam problemas semelhantes. Em muitos aplicativos de tradução para Libras a solução dada é diferente, mas ainda não é eficaz, pois apenas apresentam a palavra desejada de forma soletrada. Neste caso, apenas as letras individualmente são traduzidas para a linguagem de sinais, como mostrado pela tradução do aplicativo VLibras apresentada na Figura 3.

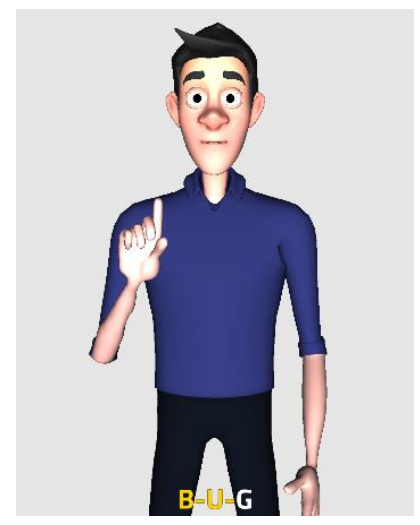

Figura 3 - Exemplo da palavra "bug" traduzida pelo aplicativo VLibras [VLibras 2018]. 
VII Congresso Brasileiro de Informática na Educação (CBIE 2018)

Anais do XXIV Workshop de Informática na Escola (WIE 2018)

Com isso, a definição dos termos em diferentes aplicativos pode apresentar divergências. Para exemplificar, foi realizado um levantamento de palavras com traduções diferentes entre o VLibras e a plataforma proposta neste trabalho. Ao menos seis palavras possuem tradução diferente: Arroba (@), Chat, Gmail, Instagram, Login e Link. Nota-se, além de palavras que possuem aplicação em outros contextos (como arroba), outras que são referentes à nomenclatura de sistemas computacionais, como Gmail e Instagram, que, na prática, podem não ser palavras contidas no dicionário da língua ou, ainda, serem termos formados por composição de outras palavras e gírias.

Assim, fica evidente a importância da contextualização dos termos traduzidos para a área em questão. Para isso, é importante o acompanhamento e supervisão de professores e especialistas do respectivo domínio de conhecimento para que não haja interpretação errônea antes mesmo de fazer a tradução para a linguagem de sinais.

Em uma análise preliminar qualitativa com dois intérpretes especialistas em Libras, eles consideraram que a proposta se mostra útil e facilita o ensino dos termos de informática aos estudantes com necessidades específicas de audição bem como contribui para o entendimento de termos da área. Além disso, afirmaram que utilizaria o dicionário novamente e também o recomendaria para seus estudantes. Foi pontuado que novos termos poderiam ser incluídos para torná-lo mais completo.

\section{Considerações Finais}

Muitos trabalhos estão sendo desenvolvidos para atender ao público com necessidades específicas. Percebe-se uma tendência da comunidade acadêmica em atender a essa demanda, mas a maioria das ferramentas disponíveis visam a sua utilização de forma genérica, automatizada na tradução de palavras para Libras e não são contextualizadas para um público-alvo específico.

O dicionário online de informática em Libras atende a um público particular para ensinar e tornar mais fácil o entendimento de termos relacionados com a área de informática. O uso da ferramenta proposta tem auxiliado o processo de ensino e aprendizagem para todos os envolvidos dentro da instituição: técnicos educacionais, professores e estudantes.

O trabalho será continuo e novas palavras da área serão introduzidas no dicionário. Com o desenvolvimento das funcionalidades colaborativas, têm-se uma interação maior com o usuário. Assim, será possível atender melhor a demanda dos usuários sobre a plataforma. A fim de alcançar uma melhor abordagem pedagógica, novos estudos sobre a utilização da plataforma e as expectativas de experiência dos usuários serão realizados.

Planeja-se efetuar uma divulgação ampla da plataforma em diversos eventos acadêmicos, para que sua utilização alcance um maior número de usuários, colaborando, assim, com um melhor ensino para os estudantes que possuem a necessidade específica da surdez. Além disso, professores das disciplinas da área de informática da instituição serão convidados a conhecer a plataforma e incentivados a utilizá-la como apoio em suas práticas pedagógicas.

\section{Agradecimentos}

Os autores agradecem às instituições PROPES/IFMT e UFU pelo apoio concedido a este trabalho. 
VII Congresso Brasileiro de Informática na Educação (CBIE 2018)

Anais do XXIV Workshop de Informática na Escola (WIE 2018)

\section{Referências}

Brasil. Lei n. 10.436, de 24 de abril de 2002. Disponível em http://www.planalto.gov.br/ ccivil_03/leis/2002/L10436.htm, acesso em 30 de maio de 2017.

Corrêa, Y., Vieira, M. C., Santarosa, L. M. C., \& Biasuz, M. C. V. (2014). Aplicativos de tradução para Libras e a busca pela validade social da Tecnologia Assistiva. In Anais do XXV Simpósio Brasileiro de Informática na Educação, SBIE’14, páginas 164-173, Dourados, MS. SBC.

Corrêa, Y., Gomes, R. D., \& Ribeiro, V. G. (2017). Aplicativos de Tradução PortuguêsLibras na Educação Bilíngue: desafios frente à desambiguação. RENOTE - Novas Tecnologias na Educação, 15(2), dezembro, 2017.

Hand Talk. Hand Talk. Disponível em https://www.handtalk.me, acesso em 20 de junho de 2018.

i.Social. Dados da Deficiência. Disponível em http://isocial.com.br/dados-dadeficiencia.php, acesso em 30 de maio de 2017.

Oliveira, L. M. B. Cartilha do Senso 2010 - Pessoas com Deficiência. Brasília, 2012. Disponível em http://www.pessoacomdeficiencia.gov.br/app/sites/default/files/ publicacoes/cartilha-censo-2010-pessoas-com-deficienciareduzido.pdf, acesso em 30 de maio de 2017.

ProDeaf. ProDeaf Web Libras. Disponível em http://www.prodeaf.net, acesso em 20 de junho de 2018.

Santos, F. T., Coutinho, F. J., \& Brito, P. H. (2016). Uma Revisão Sistemática sobre softwares educacionais para ensino de Libras. In Anais do XXVII Simpósio Brasileiro de Informática na Educação, SBIE'16, páginas 896-905, Uberlândia, MG. SBC.

VLibras. VLibras. Disponível em http://www.vlibras.gov.br, acesso em 03 de junho de 2018.

WordPress. WordPress.com. Disponível em http://br.wordpress.com, acesso em 03 de junho de 2017. 